\title{
3D sensing: Overview with a thermal non-destructive testing perspective
}

by MALDAGUE $X$.

Electrical Engineering Department, Université Laval, Québec (Québec) Canada G1K 7P4, ph: 418 /6!j6-2962, fax: 418 / 656-3594, e-mail: maldagx@gel.ulaval.ca

\section{Abstrac;t}

In this paper, we present a review of tridimensional sensing techniques which have a potential for the automatization of the thermal non-destructive testing (TNDT) task, for instance to inspect large curved specimens. The underlying ideas are two-fold. First knowledge of the shape of the inspected component allows correction of the recorded corresponding thermal images for the quantitative analysis and improved TNDT diagnosis. Second when inspecting large size components, a robotic approach to carry the TNDT head over the specimen is of interes! to save cost (higher throughput) and insure reproducibility.

The methods which are reviewed in this paper fall into two different categories: intrinsic and exirinsic. Intrinsic methods depends essentially on the infrared inspection apparatus to perform the 3D survey while extrinsic techniques rely on dedicated hardware for the 3D task. Considered intrinsic techniques are: point source heating, videothermal stereovision, direct thermogram correction and shape from heating. Considered extrinsic techniques are: stereopsis, photometric stereo, shape from shading, texture, motion, occluding contour, optical flow, moire, focusing, active triangulation, time of flight and shape from spatial encoding. These techniques will be briefly reviewed. Other extrinsic techniques have been developed but seem to have less interest for TNDT applications and were not included in this review although an annotated bibliography is included at the end of this paper and presents a mix of 3D sensing techniques and developments. Considerations on diffractive optical elements for structurad light projection are included as well as a case study illustrating the use of robotic in a TND'T inspection system.

\section{Introduction}

Use of thermal non-destructive testing (TNDT) is spread in different fields of applications and industrial contexts (see references [1], [2], [3], [4], [5], [6] and other papers in these proceedings for general information about TNDT itself). Moreover, the recent availability of both advanced infrared cameras with greater spatial and temperature resolutions such as those based on focal plane array sensors (FPA) as well as powerful and affordable powerful workstations open new opportunities to TNDT in terms of thermogram quality and processing. Traditional qualitative interpretation can now be replaced by a complete quantitative analysis. In this respect, the first task in TNDT is signal acquisition, and the problem we will address in this work is the question of sensing of non-planar - tridimensional (3D) - surfaces in a TNDT perspective.

The purpose of this work can be seen with two different points of view. First, as it will be shown, availability of $3 D$ information relative to inspected components open the door to thermogram corrections of possible distortions due to object curvature in order to increase reliability of the TNDT diagnosis. For instance, fusion of information between recorded thermal data, part shape and thermal modeling leads to an increased reliability of the TNDT diagnosis [7], [8].

The second motivation is more technological and economical. "Robots" taken in a broad sense use are now coming in many fields: improved cars, kitchen and telecommunication aids, etc., NDT. In NDT robots or automated manipulators are now in use in order to maximize 
inspection throughput and reproducibility [9]. For instance, it is reported that $80 \%$ of the 10 -day cost related to C-type ultrasonic inspection of an aircraft is related to the immobilization time [10]. Since one of the strong point of TNDT is high inspection rate (field rather than point scanning as in ultrasonic NDT for instance), it is natural to combine this NDT technique with robotics. In such applications, the NDT inspection head is mounted directly over the robot-enddefector.

At this point two approaches are possible: one is preprogrammed surveys [9], the other is autonomous navigation over the specimen to inspect [11]. In the first approach, the robot is taught so that it will move over the surface to inspect in a blind fashion, regardless of possible misalignments. This approach is convenient if the same task is to be repeated over a large batch of identical parts mounted in the exact same position on some fixing rack (such as at the production level). In the second approach, the robot will sense and determine by itself the path to follow to inspect the surface. This approach is convenient if little or nothing is known a priori about the surface to inspect and it is more oriented for maintenance inspection tasks.

Such automatic navigation and thermogram correction tasks require knowledge of component curvature. To obtain this information, it is required to perform some 3D measurements. In this paper, an overview of the current 3D sensing techniques will be given with particular emphasis to those having a potential use in TNDT. Only the more attractive techniques in the context of TNDT will be discussed for instance, radar, fresnel diffraction, holography, shape from line drawing, will not be covered. At the end of this paper, a selected annotated bibliography is included. Interested readers will find there additional information in the field of $3 D$ sensing. See also the following books from which some of the material presented here is extracted [12], [13], [14], [15], [16].

\section{Preliminaries}

A range image $r(i, j)$ contains, for every pixel at coordinates $(i, j)$, the corresponding distance $r(i, j)$ between a surface patch on the object in the scene and the ranging system. This is the main difference with respect to a conventional video image where pixel values represent brightness. In an orthographic projection, where light rays from the object are assumed parallel one to each other and perpendicular to the image plane, the following affine transformation expresses the absolute cartesian coordinates $(x, y, z)$ of a given pixel value located at the $i$-th row and $j$-th column of the range image:

$$
\begin{aligned}
& x=a_{x}+s_{x} i, \\
& y=a_{y}+s_{y} j, \\
& z=a_{z}+s_{z} r_{y},
\end{aligned}
$$

where s-values are scale factors (distance increments) and a-values are coordinate offsets.

Orthographic projection is valid when viewed objects are remote. At close distances, it is better to use a perspective projection. In this case, a spherical coordinate system is used and the camera is seen as a pin hole: only light rays coming from the object through the pin hole fall on the image plane and the $(i, j)$ indices correspond to angular increments in the vertical $\phi$ (polar, elevation) and horizontal $\boldsymbol{\theta}$ (azimuth) directions, then (Figure 1 ):

$$
\begin{aligned}
& x=a_{x}+s_{r} r_{l y} \cos \left(i s_{\phi}\right) \sin \left(j s_{\theta}\right), \\
& y=a_{y}+s_{r} r_{l y} \sin \left(i s_{\phi}\right), \\
& z=a_{z}+s_{r} r_{I y} \cos \left(i s_{\phi}\right) \cos \left(j s_{\theta}\right) .
\end{aligned}
$$

Ranging sensors can acquire data in a point, a line, a full-field scheme or by fusion of multiple views. This last scheme is particularly useful when surface points of interest are not visible or cannot be measured from a single viewpoint. In TNDT applications we are mostly interested to line or full-field range measurements which can match thermal image format. 


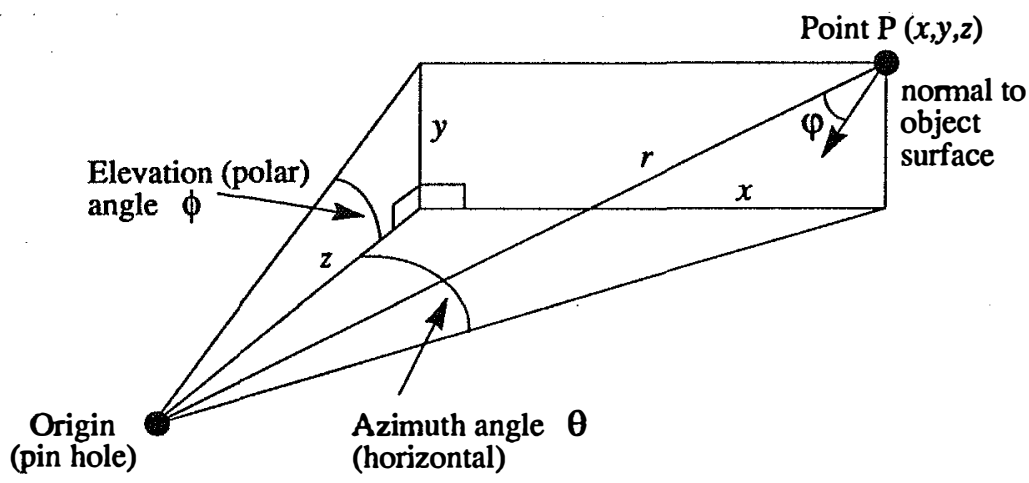

Fig. 1 - Definition of the system of coordinates.

Lateral motion (of the robotic arm) associated to a line sensor can provide complete scanning of the inspected component.

As characteristics of 3D sensing systems (3DSSs), resolution, repeatability and accuracy are important issues to address in addition to scanning rate, weight, case sizing, range of operation, cost, etc. Range resolution is the smallest change in range that a 3DSS can detect; range repeatability is a measure of the statistical deviation when the same distance is measured over time; range accuracy is a measure of the statistical deviation when a knownvalue distance is measured over time. Normally, a 3DSS with good repeatability could be calibrated to be accurate.

With respect to TNDT, 3DSSs can be divided into intrinsic and extrinsic classes. Intrinsic techniques make little use of extra methodologies besides the TNDT task itself to obtain distance information (or at least object surface orientation or shape). On the other hand,extrinsic techniques rely completely on non-TNDT hardware to extract the ranging informations.

\section{Intrinsic 3D sensing}

Active extrinsic 3DSSs rely on energetic illumination devices such as laser beams (see paragraph 5, below). The large number of photons collected by the sensor from the illumination device allows to achieve great performances. Since intrinsic 3D sensing relies mainly on the TNDT apparatus and on the poorly energetic thermal radiation to extract object shape, it is not surprising that reported accuracy and resolution are limited. However, appropriate reconstruction processing alleviates partly this problem. A review of these techniques can be found in [17]. In this section we will only recall the main features of these techniques. Generally they require processing of an early recorded thermogram (ERT) in which potential defect contrasts have not yet developed. Following the analysis of the ERT, the whole thermogram sequence can be corrected for surface curvature.

\subsection{Point source heating correction}

This technique makes use of an omni-directional heating source to thermally stimulate the specimen (for information about active transient TNDT scheme, refer for instance to [5], p. 6). This heating source can be approximated by a point source for which the irradiance (i.e. the energy falling on a surface, [18], p. 203) depends on the square of the distance $r$ between a given surface patch $d A$ and the point source. Hence, the surface temperature rise $\Delta T$ on the surface patch $d A$ (after a given exposure time $t_{i}$ ) can be expressed by:

$$
\Delta T=\frac{a_{1} \cos \varphi}{r^{2}}
$$


http://dx.doi.org/10.21611/qirt.1994.031

where $a_{1}$ is a calibration constant and $\varphi$ is the angle between the normal to surface patch $d A$ and the direction to the point source. If parameters $a_{1}$ and $\varphi$ are available, it becomes obvious that range information $(\lambda)$ can be extracted from temperature measurement $(\Delta T)$. Surface orientation expressed here by angle $\varphi$ depends in fact on two gradient parameters as explained in paragraph 4.2. However for surfaces having $\varphi \sim 0^{\circ}$, and then $\cos \varphi \sim 1$, the problem is simplified (single dependence on $a_{1}$ ). A powerful light bulb mounted over the infrared camera can serve for such purpose (Figure 2). Proper calibration of the temperature

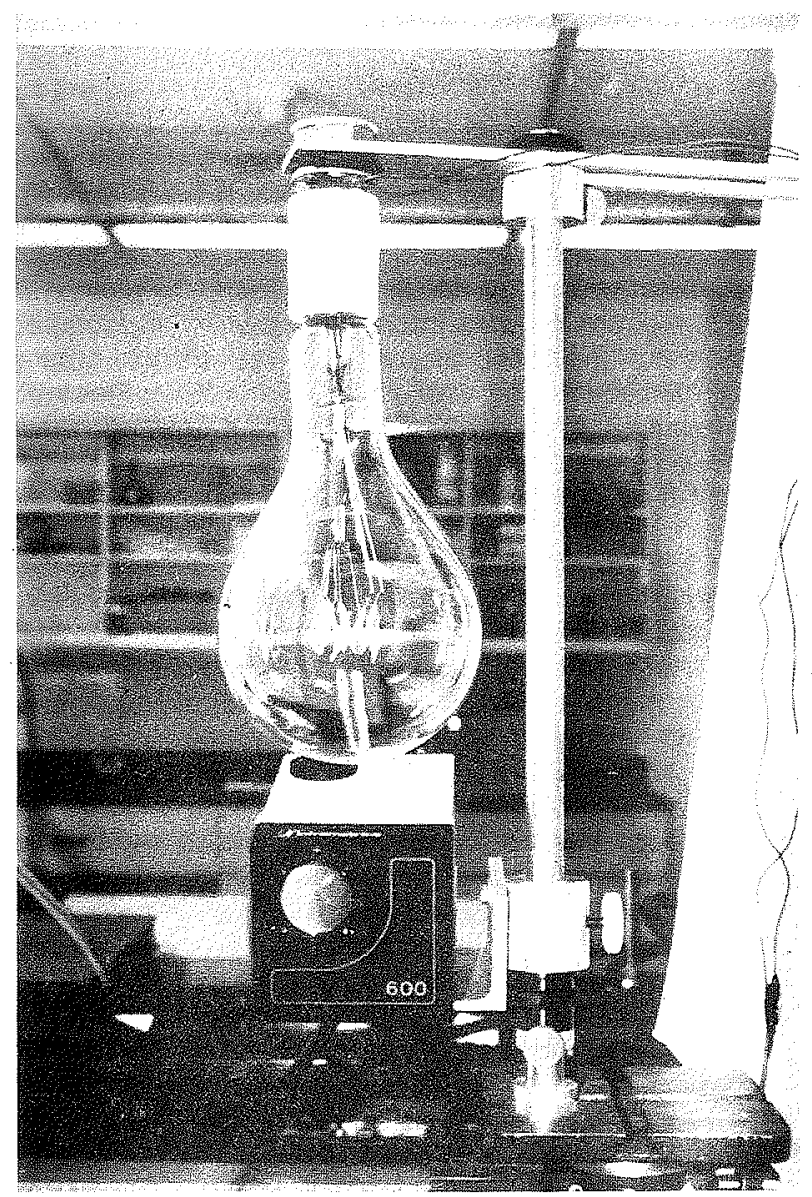

Fig. 2 - Heating apparatus for point source heating correction.

rise, deposited in the volume in front of the infrared camera-point source assembly, is possible by shifting a plane at different locations along the optical axis. Range informations is obtained by direct look-up and interpolation with calibration data. Although demonstrated (at least from a laboratory point of view), there are many problems associated with this technique: the angle $\varphi$ is neglected, the resolution is poor in the reported demonstration $(1 \mathrm{~cm}$ for a $20 \mathrm{~cm}$ range in front of the infrared camera, for a $1000 \mathrm{~W}$ lamp), the heating efficiency is weak since the point source emits in all directions, while calibration drifts including lamp aging further degrade repeatability. 
http://dx.doi.org/10.21611/qirt.1994.031

\subsection{Video thermal stereovision}

This method is based on the registration of two images, one recorded in the visible spectrum from which local surface orientation information is obtained and one being the considered thermogram to be corrected for orientation distortions. Since two cameras are involved, we talk of stereovision although the method differs from the conventional stereopsis method exposed in paragraph 4.1. Under some hypotheses (Lambertian opaque surface), pixel intensities $L$ of the visible image can be expressed by:

$$
L=K \cos \varphi+C
$$

where $K$ and $C$ are the calibration constants of the visible imaging systems and $\varphi$ is the local surface orientation.

Once calibration is done (on two different objects of known orientation) and after proper registration between both images (due to the different points of view for the visible and thermal images), it becomes possible to extract local orientation $\varphi$ from the visible image and apply the correction $(\cos \varphi)$ on recorded thermograms following equation (3). The main hypotheses made here is that the heating has a little dependence on distance (the dependence being more on the local orientation $\varphi$ of the surface) while both the emissivity in the infrared and the reflectance in the visible spectrum being high. Besides these restrictions and the additional hardware required, this correction method for local surface orientation is efficient.

\subsection{Direct thermogram correction}

This is a variation of method of paragraph 3.2 where the extra hardware (visible camera + its illumination device) is suppressed and where equation (4) is directly applied on the ERT to extract local surface orientation $\varphi$ which is subsequently used to correct the sequence of thermograms. As for method of paragraph 3.2 heating should have little dependence on distance.

\subsection{Shape from heating}

This appellation was given to this method due to some common similarities with shape from shading method as for instance its ability to restore shape but not absolute distance measurements (see paragraph 4.2, below). This method relies only on the TNDT apparatus to extract local surface orientation. The following hypotheses apply. The assumed projection is orthogonal, the heating flux is assumed orthogonal to the specimen (if considered flat) and the heating has a little dependence on distance (the dependence being more on the local orientation $\varphi$ of the surface). The ERT is analyzed row per row. Each row is divided into segments, either linear or non-linear. For linear segments, local orientation $\varphi$ is given by:

$$
\sin \varphi=\frac{\Delta T}{S d}
$$

where $\Delta T$ is the temperature variation between segment extremities, $d$ is length of the segment (related to the number of pixel of the segment with a factor of scale due to the orthographic projection used) and $S$ is the slope of the curve: temperature rise versus distance in front of the heating apparatus. In a first calibration procedure, parameter $S$ can be obtained using equation (5).

For non-linear segments, the local temperature maximum $T_{\max }$ of the segment is found and knowing initial, before heating temperature $T_{1}$, local orientation is given by:

$$
\cos \varphi=\frac{T-T_{i}}{T_{\max }-T_{i}}
$$

Corrected segments are next merged together to establish the complete shape along the analyzed row. An additional procedure is used to take into account the non ideal line spread 
http://dx.doi.org/10.21611/qirt.1994.031

function of the infrared camera to restore abrupt shape discontinuities. This method is based on an ellipsoidal noise model.

In terms of performance and relative simplicity, this is probably the more interesting intrinsic 3D sensing method reviewed here. Reported accuracy is in the order of $5^{\circ}$ and $5 \mathrm{~mm}$.

\section{Extrinsic passive 3D sensing}

In the next two sections, we will review dedicated 3DSSs for extraction of ranging information. In section 4, techniques which do not rely directly on dedicated illumination are discussed.

\subsection{Stereopsis}

This broad family of 3DSSs relies on ambient lighting. Stereopsis is based on two 2D sensors to obtain ranging information (as for the human visual system). Once correspondence between the two images is established, 3-D coordinates of a given surface point $P(x, y, z)$ are computed as follow (Figure 3):

$$
(x, y, z)=\frac{L}{g}(u, v, f)
$$

where $L$ is length of the baseline, $f$ the focal length and $(u, v)$ are two-D coordinates of point of interest as seen by the $2 \mathrm{D}$ sensors.

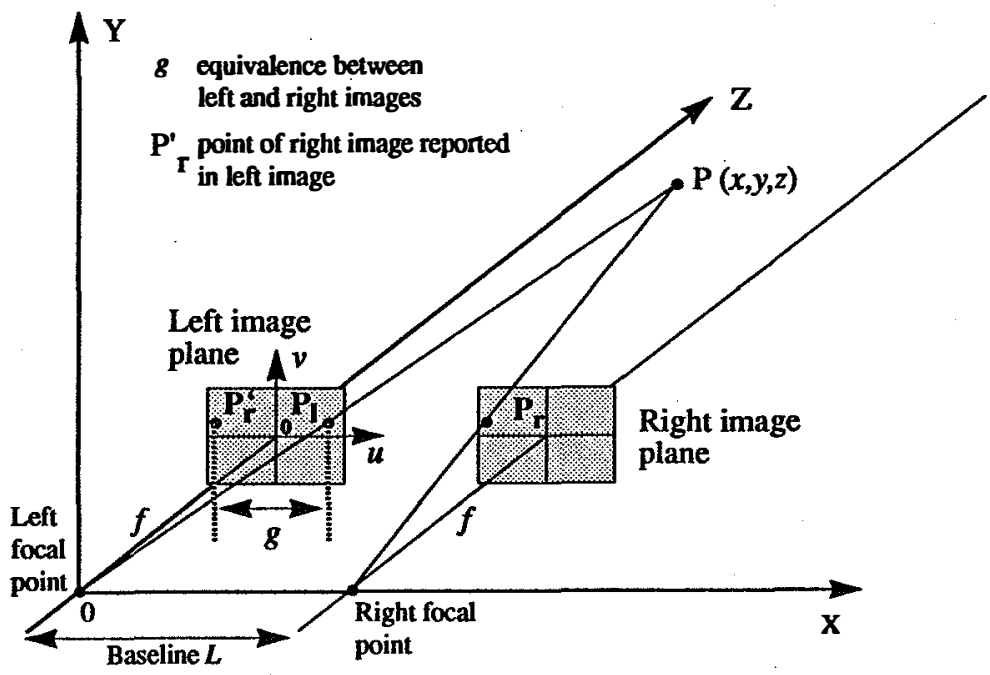

Fig. 3 - Configuration for stereopsis 3D sensing (adapted from [14], fig. 2.1, p. 68).

Problems of this technique are the complexity to match corresponding points in the two images, and the occlusions and shadows due to the non-null baseline between the two sensors which may prevent full view of the scene from the two different point of views. One eye stereo solves these problems but the task to synthetize the second (virtual) image is complex. Photometric stereo (see paragraph 4.2) solves this problem by using one camera and two (or more) different illumination conditions. For TNDT applications hindrance of stereopsis can be a concern, especially if mounting on a robotic manipulator is envisaged. 
http://dx.doi.org/10.21611/qirt.1994.031

\subsection{Photometric stereo, shape from shading, and texture}

In shape from shading, shape extraction in a 2D image is based on the relationship between surface orientation and reflected light intensity or image brightness. The (visible) image from an object is a function of its shape, its reflectance properties and the distribution of light sources. The reflectance properties for an object are expressed (Figure 4-a) by the bidirectional reflectance distribution function (BRDF) relating the brightness of a surface to the viewing direction $\left(\dot{\theta}_{\theta}, \phi_{\theta}\right)$ and to the direction of the light source $\left(\theta_{1}, \phi_{i}\right)$. For example, in the case of a Lambertian surface appearing equally bright in all directions, the BRDF is $1 / \pi([18]$, p. 209). The reflectance map relates explicitly surface orientation to brightness by encoding information on surface reflectance properties and light source distributions. A surface in the space can be described by its perpendicular distance $z$ from a reference plane $X-Y$.

(a)

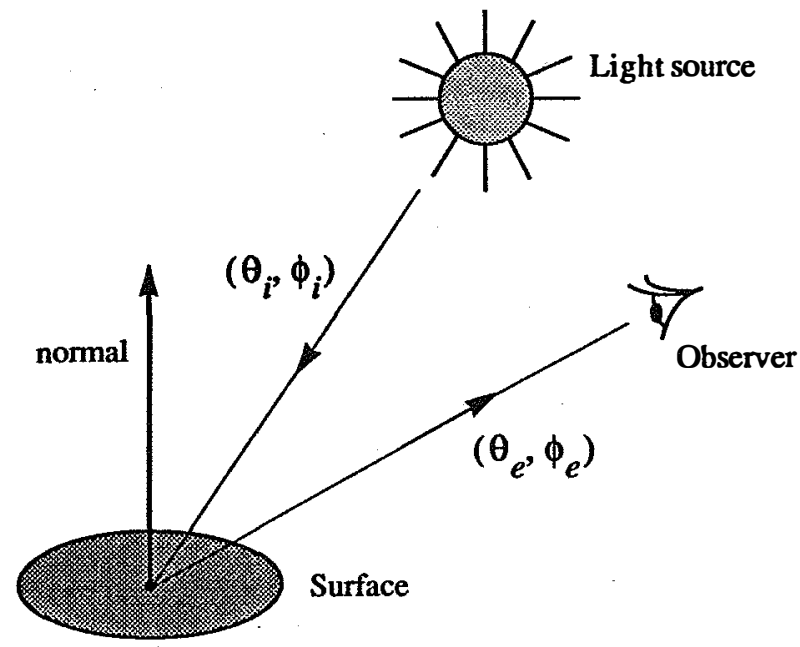

(b)

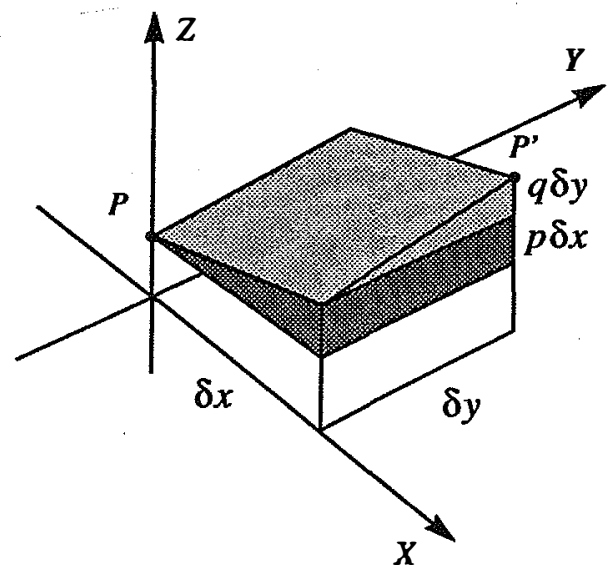

Fig. 4 - (a) Reflectance properties of a surface, (b) surface orientation expressed by partial derivatives $p$ and $q$ (adapted from [18], fig. 10-9, p.214 and fig. 10-11, p.217).

Considering a small step $\delta x$ in the $x$-direction starting from a given point $P$, change along $z$ direction can be expressed by ( $P^{\prime}$, Figure 4-b):

$$
\delta z=\frac{\partial z}{\partial x} \delta x+a
$$


http://dx.doi.org/10.21611/qirt.1994.031

where a regroups higher terms of this Taylor series development ([18], p. 217). Abbreviations $p$ and $q$ are used respectively for $\delta z / \delta x$ and $\delta z \delta y$ respectively. The reflectance map $R(p, q)$ is thus defined in the gradient space $(p, q)$ and is generally represented by iso-brightness contours. The reflectance map $R(p, q)$ associates to a surface point of orientation $(p, q)$, a given brightness value. Using the image-irradiance equation, recovering $3-D$ shape from shading information is possible with:

$$
R(p, q)=E(x, y)
$$

where $E(x, y)$ is the brightness value at point $(x, y)$ and $R(p, q)$ expresses orientation. The problem with equation ( 9$)$ is that an infinite number of surface orientations corresponds to a given brightness (an iso-brightness contour on a reflectance map plot connects such set of orientations). Additional information is thus required to recover local surface orientation.

- Photometric stereo makes use of illumination from two (or more) different illumination directions to solve this problem, two equations $R_{1}(p, q)=E_{1}$ and $R_{2}(p, q)=E_{2}$ allow to determine the two unknowns $(p, q),[19]$.

It is also possible to recover shape from a single image as for instance when people look at pictures in magazines and are able to recognize faces. Some constraints such as smoothness (lack of discontinuities in depth) allow to growth solutions along paths (known as characteristic curves) over the analyzed surface.

For example, assuming a linear reflectance map, $R(p, q)=f(a p+b q)$ giving rise to an image $f^{-1}(E(x, y))=a p+b q$ and taking a direction $\theta_{0}$ on this observed surface, the slope $m$ can be written: $m(\theta)=p \cos \theta+q \sin \theta$. Now, starting at a particular point in the image and making a small step of length $\delta \varepsilon$, producing a change in $z$ of value $\delta z=m \delta \varepsilon$ and integrating this last equation yields to ([18], p. 247):

$$
z(\varepsilon)=z_{0}+\frac{1}{\sqrt{a^{2}+b^{2}}} \int_{0}^{s} f^{-1}(E(x, y)) d \varepsilon
$$

- As seen in equation (10), although absolute distance can not be recovered (the integration constant $z_{0}$ is unknown), the shape (that is the variations in depth over the surface) is determined. This is the principle of the shape from shading method.

- Texture is also a good cue for surface orientation. Notice for instance the sizes of bricks on a brick wall view from the side: the relative range as seen from the viewer is coded in the diminishing brick size. In order to estimate depths from texture, the texture gradient must be computed.

All these techniques (photometric stereo, shape from shading and texture) are based on a well-established background and are thus interesting 3D sensing methods. However, even today they are still seen as "sophisticated methods" while their practical use on the plant floor is not direct, especially in the case of parts of complex geometry, in presence of spurious and specular reflections, of light sources of unknown orientations and of textures of uneven sizes. Besides shape from heating discussed previously, these ranging techniques have not yet seen direct use in conjunction with TNDT. Texture can be interesting, especially in the case of CFRP components exhibiting distinctive web surface patterns.

\subsection{Structure from motion, occluding contour and optical flow}

These techniques have some interest for specific applications. For instance if robotic displacement of the TNDT head over the inspected surface is envisaged as discussed later in paragraph 6. In structure from motion, the observation of a number of points in two or more views allows to determine the tridimensional coordinates of these points (see for instance work of [20]). Potential problems with respect to TNDT are the relative large size of inspected objects which complicates the correspondence procedures between the different views, especially the presence (or lack) of distinctive landmarks. The same discussion applied to the shape from occluding contours approach for which the basic idea is borrowed from shadow 
http://dx.doi.org/10.21611/qirt.1994.031

graphs where a person's finger may be arranged to produce shadows of different 3-D shapes such as animals [21].

In shape from optical flow, the field of apparent velocities of points on the surface of objects moving in space is used to estimate object structure (symmetrically, a ranging sensor moving over a stationary surface can be used instead). The observation in time of a small but finite textured surface patch moving in space allows to derive deformation parameters which are related to 3-D surface structure. With respect to TNDT applications, potential problems are the difficulty to locate such textured surface patch on the inspected component (think to a large smooth curved aircraft component exhibiting no specific landmark for instance) and also the (yet) limited spatial resolution of infrared cameras.

\section{Extrinsic active 3D sensing}

In active 3DSSs, a special lighting is used to illuminate the scene.

\subsection{Moiré}

As it is well known and observed, a grating projected onto an object deforms according to the 3D shape of the object, this is the principle of Moire contourgraphy. A Moire pattern is obtained by superposing two gratings with regular space patterns of high spatial frequency which produce a low spatial frequency interference pattern, Figure 5. These Moire fringes carry information about surface shape $(\Delta z)$ which can be extracted once fringes are located in the image. Angular separation of source and detector is also critical to range measurements while inspected surface can not exhibit depth discontinuities. Although relatively inexpensive to implement, problems in Moiré are related to the difficulties to achieve accurate calibration and automated analysis of fringes (i.e. tracking of fringe ridges and valleys). With respect to TNDT, as for other 3DSSs where illumination is involved, requirement for high emissivity in the infrared can be a problem due to the need for a good reflectivity in the visible.

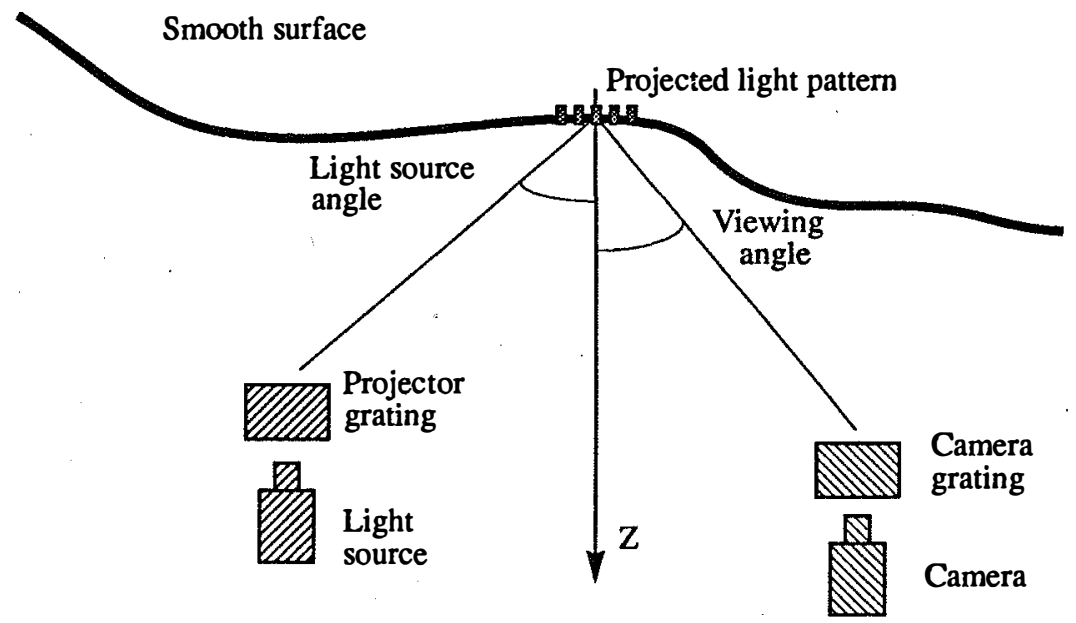

Fig. 5 - Moire $3 D$ sensing by the pattern-projection method (adapted from [14], fig. 1.14, p.35 and [25], fig. 5-36, p.304).

\subsection{Focusing}

Focusing ranging techniques are based on the thin lens equation which stipulates the distance $z$ from the lens of focal distance $f$ at which the observed object will be at focus on the image plane located at a distance $w$ :

$$
\frac{1}{w}+\frac{1}{z}=\frac{1}{f}
$$


http://dx.doi.org/10.21611/qirt.1994.031

Light passing through a lens of finite aperture (diameter $D$ ) experiences diffraction (blur). The radius of blur for a point located in $z$ is minimum when in focus at $w_{\text {focus }}$ and increases when $w$ moves away from $w_{\text {rocus, }}$ in either direction. Modeling this blur by a 2D Gaussian function of size $\sigma$, gives the range for this point:

$$
z_{ \pm^{\prime}}(\sigma)=\frac{w f}{w-f \pm \sigma F}
$$

with $F=f / D$ (f-number of the lens aperture).

If the object is moved from this position, the object image will be blurred and the degree of this blur can be related to range information.

Commercial systems are available such as the BIRIS camera which is robust, compact and reliable since containing no moving parts. Perspectives in TNDT are interesting for this kind of 3DSSs, specially for robotic inspection as discussed previously (paragraph 1). Figure 6 shows the defocusing principle used in the BIRIS camera ([22]). It is made with a CCD camera with a mask and uses a light source (laser diode) to project a point on the scene. A mask with two small apertures is mounted in front of a standard CCD camera. The camera is focused such that the point $A$ on the reference plane is in focus in $A^{\prime}$ on the sensor. The point $B$ on the object located in front of the reference plane is in focus in $B^{\prime}$, behind the image plane and due to the mask, two points $\left(b_{1}\right.$ and $\left.b_{2}\right)$ are imaged on the sensor. It can be demonstrated that distance $\left(b_{1}-b_{2}\right)$ is related to ranging coordinate $z$ of point $B$ while geometrical center of $b_{1}$ and $b_{2}$ gives the $x$ and $y$ coordinates. The main problem with this defocusing technique is the limited separation between the two apertures of the mask (limited by the lens aperture itself) and thus the separation between $b_{1}$ and $b_{2}$ is restricted while $\left(b_{1}-b_{2}\right)$ must be evaluated at subpixel accuracy to have a good resolution in depth.

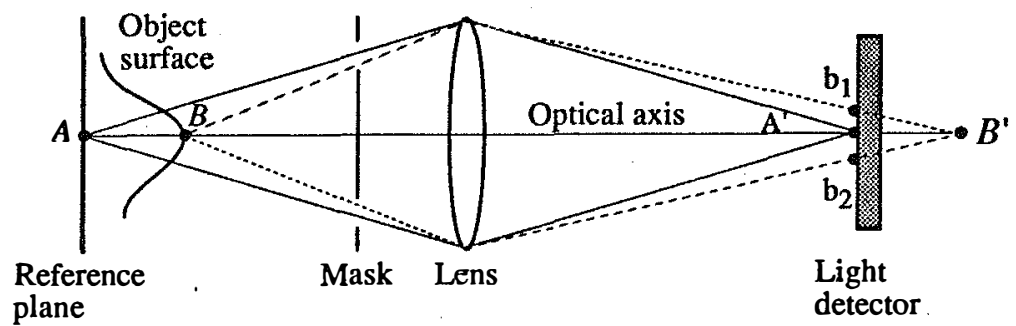

(a)

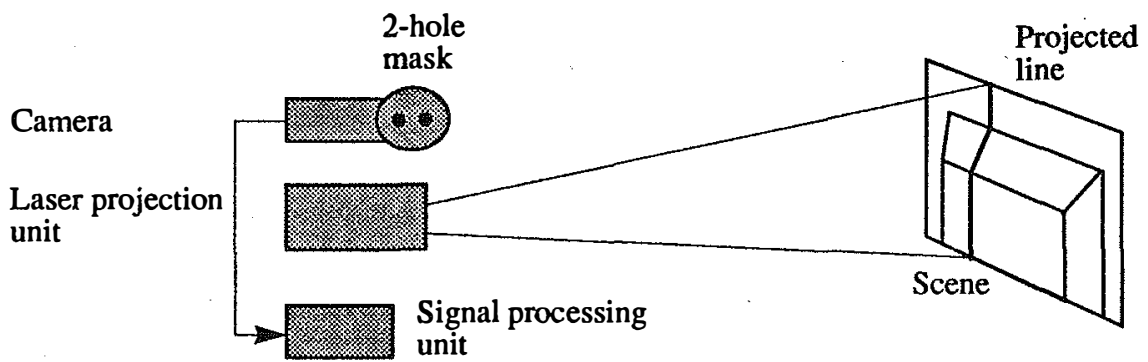

(b)

Fig. 6 -(a) 3D sensing from defocusing (BIRIS camera principle), (b) apparatus configuration (adapted from [22]). 
http://dx.doi.org/10.21611/qirt.1994.031

\subsection{Active triangulation}

This broad family of techniques involves at its basis, the law of sines which stipulates that, if the length $B$ of one side (the baseline) and the two corresponding angles $\angle A B$ and $\angle B C$ of triangle $A B C$ are known, the triangle is completely defined, that is sides $A, C$ and angle $\angle A C$ can be obtained from:

$$
\frac{\sin (\angle A B)}{C}=\frac{\sin (\angle B C)}{A}=\frac{\sin (\angle C A)}{B}
$$

In active triangulation methods, some sort of lighting is projected onto the scene and ranging is computed from accurate measurements of angles and baseline. Figure 7 illustrates a basic system: where a light projector illuminates a point $P(x, y, z)$ onto the surface and is imaged on pixel $(u, v)$ onto the camera sensor. With respect to Figure 7 , similar triangles give (with $f$ the focal length): $u z=x f$ and $v z=y f$ and thus measurement of $(u, v, \beta)$ yields to computation of tridimensional coordinates $(x, y, z)$ :

$$
\begin{aligned}
& x=u \frac{b}{f \cot \beta-u} \\
& y=v \frac{b}{f \cot \beta-u} \\
& z=f \frac{b}{f \cot \beta-u}
\end{aligned}
$$

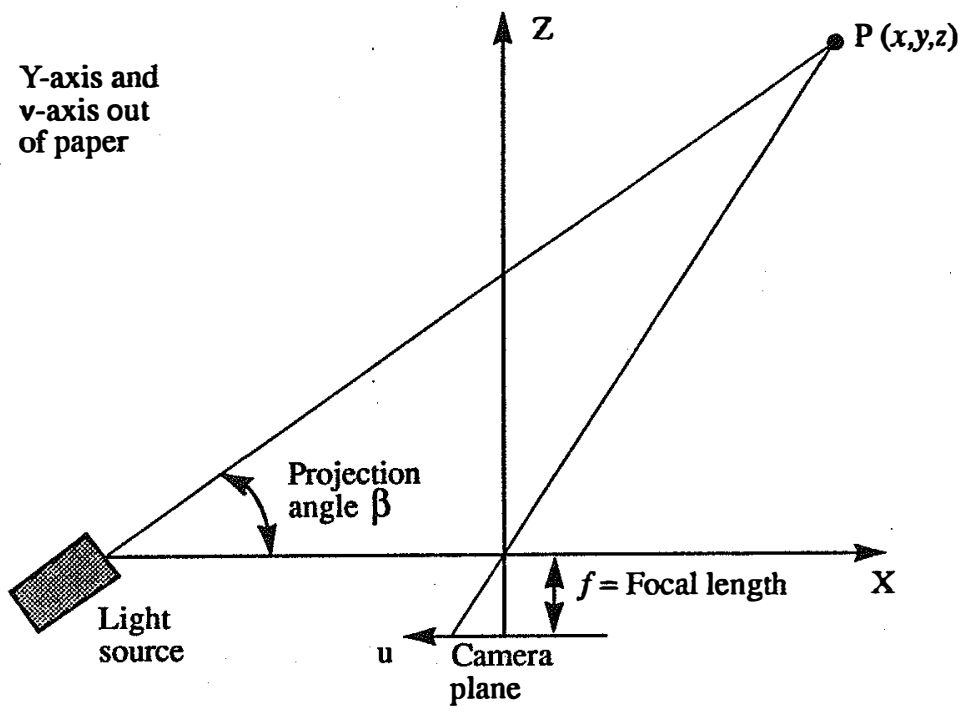

Fig. 7 - 3D sensing by active triangulation. (adapted from [14], fig.

Many variations of this principle have been developed using different projection schemes: line, multiple points, multiple lines, grid, circle(s), cross, thick stripes, coded binary patterns, color-coded stripes and random texture. In many cases, to measure range in the whole scene, some sort of scanning mechanisms are needed (e.g. scanning or rotating mirrors or galvanometers) and may add to the cost, bulkiness, limited reliability, calibration requirements. In the case of system of Figure 7, such scanning mechanism provides parameter $\beta$ directly. For instance, in the case of a galvanometer onto which a small mirror is attached and is used to deviate a laser beam, value of the electric current crossing the galvanometer is directly 
linked to deviation angle $\beta$. One of the problem of conventional triangulation system i:; that a large baseline separation between the source and the camera is preferred with riossible shadow effects (some points can not be seen/illuminated simultaneously), moreover, large baselines imply a larger camera head which is not convenient (e.g. this may restris:t robot displacement due to encumbrance).

Some active triangulation techniques have emerged from the labs into commercial products. One of such products is the ranging camera line from Servo-Robot (Jupiter and Saturn models) These ranging cameras are based on synchronized triangulations which overcome the large baseline requirement without penalty on resolution and field of vie:w [23], [24]. In this design, projection and detestion are synchronized by means of a pyramidal rotating mirror (Figure 8). The beam leaves the source and illuminates point $P(x, y, z)$ thiough a fold path on mirrors $S$ and $M 1$. This illuminated spot is viewed on the opposite side of the mirror $S$ by reflection on mirror $M 2$. This system allows to retrieve ranging information along a line on the inspected surface. Addition of a vertical nodding mirror $N$ permits to cover a full surface. Tridimensional coordinates $(x, y, z)$ are determined by scanning mirror angle $\beta$, vertical nodding mirror angle $\alpha$ and horizontal position detector value $p_{\mathrm{d}}$. The small distance between projection and detection facets of mirror $S$ limits shadow effects. While convधntional triangulation such as the one depicted on Figure 7 requires 2-D imaging dietector, synchronized triangulations require only a linear sensor (such as a CCD strip) thus incseasing the bandwidth (from a few $\mathrm{KHz}$ to $\mathrm{MHz}$ ). For instance a $128 \times 256$ range image is created in less than $1 \mathrm{~s}$, the spatial resolution being less than $1 \mathrm{~mm}$ in $x, y$ and $z$.

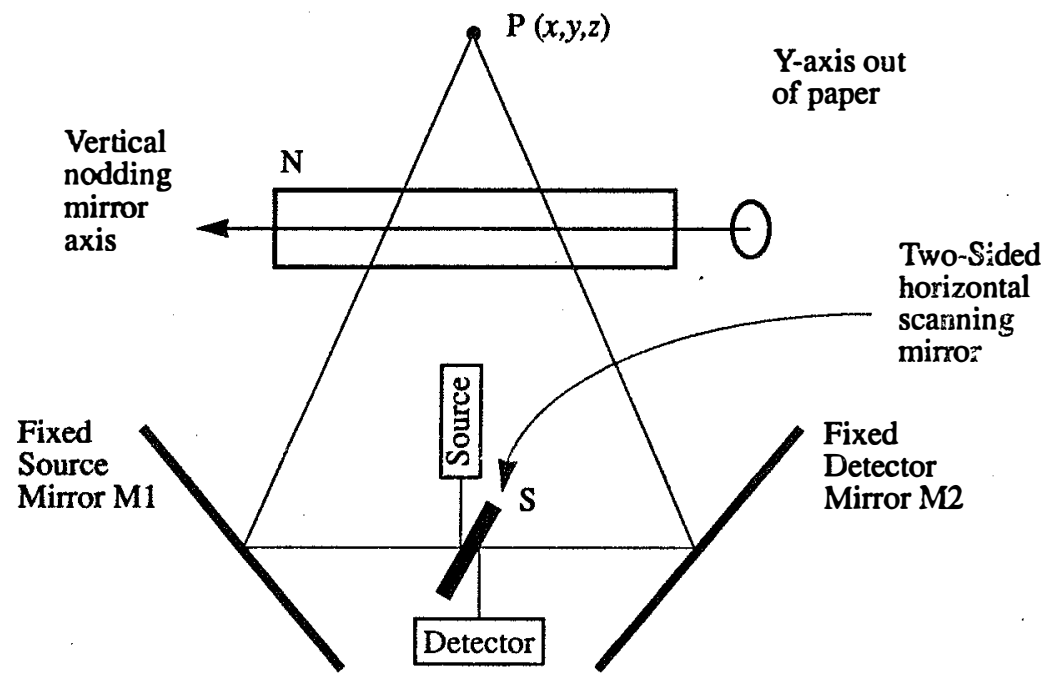

Fig. 8 -3D sensing by synchronous scanning (adapted from [23] and [24]).

Maintaining depth of focus for all depths in triangulation systems for both illumination and detection can be a problem. The Scheimpflug condition ([14], p. 25) stipulates that, to maintain focus over all depths, detector plane must be tilted with respect to the viewing direction. For instance the synchronized system of Figure 8 has an optimal focus on a spherical reference surface thus limiting the angular displacement over which the scanned spot is well focused. Furthermore, the translation speed of the flying spot over a flat surface is slightly higher on the edges than on the central position of the scanned path for a constant mirror rotation rate, this is a source of errors when a precise metrology of the scanned surface is required ([25], $p$. 150).

Synchronized 3DSSs such as those of the type of Figure 8 are particularly well adapted to industrial machine vision and to robotic TNDT in particular due to their compactness (as discussed below in paragraph 6). 
http://dx.doi.org/10.21611/qirt.1994.031

\subsection{Time of flight techniques}

There exists many different variations of this technique such as radar, ultrasonic and laser time of flight (tof). Since radar tof is primarily used for airborne surveying such as in planetary space exploration or for hydrographic surveys, it will not be discussed here.

Ultrasonic tof is based on the following relationship:

$$
2 r=v_{u} t_{f}
$$

where $v_{u}$ is the speed of sound under the considered conditions (of humidity, temperature and pressure), $t_{f}$ is the time of flight of the pulse that travels over distance $2 r$ during its round trip between the object surface and the transmitter/receiver unit. Since the baseline of ultrasonic tof sensors is null, there is no shadow problem as discussed in the case of some other 3DSSs. On the other hand, it is difficult to obtain a well-focused ultrasonic beam and consequently, "ultrasonic images" exhibit a poor resolution. Another problem of ultrasonic tof is that at some incidence angles only little energy is reflected back and the signal is lost in the detector noise.

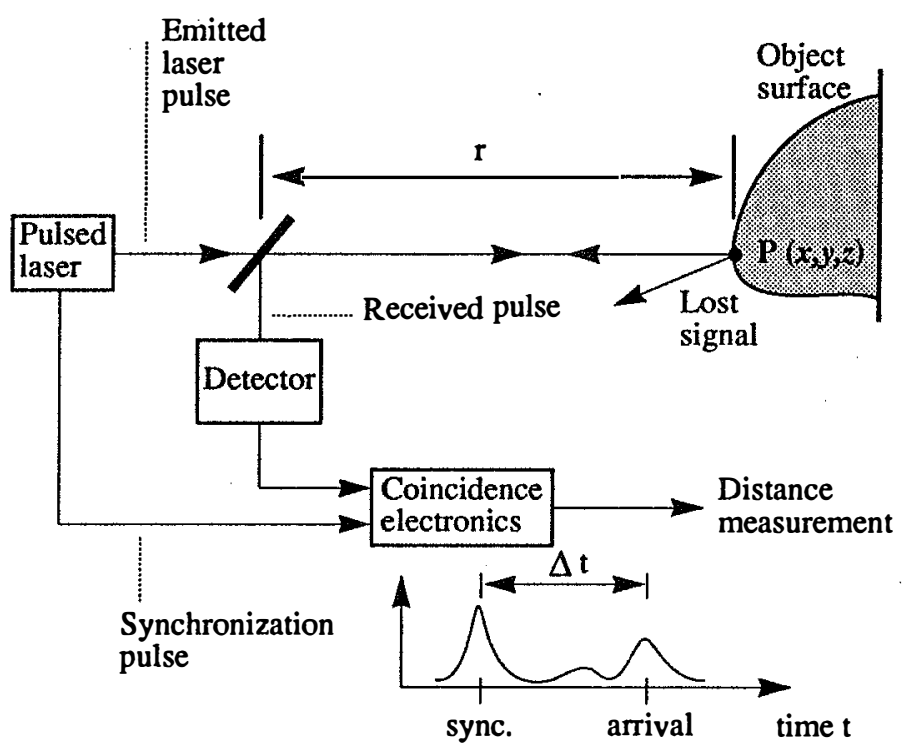

Fig. 9 - 3D sensing using a laser time of flight device (adapted from [14], fig. 3.12, p. 145).

In laser time of flight, a laser replaces the ultrasonic source. Figure 9 illustrates the principle of operation. A laser pulse is transmitted on the scene through a semi-transparent mirror, impinges on the surface on $P$ and reflects back to a detector. The time interval between the starting synchronization pulse (generated simultaneously with the laser pulse) and the arrival pulse (generated with the detection of the reflected back pulse) is directly related to the distance between the surface point $P$ and the pulsed laser as in equation (15). Since a laser beam is well focused (as compared to an ultrasonic beam), a better resolution is achieved while the null baseline provides a shadow-free method. There are two main problems associated with laser tof. Firstly, very fast electronics is needed to accurately measure short time intervals (a $2 \mathrm{~m}$ range yields to a tof of about $15 \mathrm{~ns}$ while a $\pm 5 \mathrm{~mm}$ accuracy implies an accuracy on tof measurement of about $\pm 15 \mathrm{ps}$ ). Secondly, in some cases, the returned power on the detector (often a photomultiplier tube or a signal intensifier device) may be very low thus yielding to erroneous measurements since the signal is drown in the noise. In phase laser tof, the laser beam transmitted is amplitude modulated with a sinusoidal waveform and the phase of the reflected received signal is proportional to the tof (round trip) and thus to the 
http://dx.doi.org/10.21611/qirt.1994.031

distance. Although these phase laser tofs solve the fast electronics requirement of the conventional laser tofs, they are slower.

\subsection{Shape from spatial encoding}

In this technique, a structured lighting (often some sort of "grid") is projected on the scene and the pattern of illumination is interpreted in term of surface geometry. The underlying idea is that the use of spatial encoding reduces the computational complexity, while it improves the reliability. This is a suitable technique in laboratory conditions where environment (lighting) can be controlled, although new developments emerge [26].

\subsection{Diffractive optical elements for structured light projection}

Active $3 D$ sensing is sometimes criticized because structured light projection involves some sort of mechanical scanning devices prone to possible failure and lack of ruggedness. An altemative to the traditional projection method, based on a mirror attached to an oscillating galvanometer to deviate the laser beam and project points and line, is to rely on diffractive optic elements. Dammann gratings were introduced in 1971 [27] and are now commercially available (for instance by Lasiris, Montréal, Canada). Basically, they are made of many identical cells inducing phase transitions in the transmitted beams (the diffracted light produces a large number of beams which interfere together to produce a pattem). The difficulty is to adjust the positions of the transitions to obtain a given energy distribution in the various outputted beams. An important characteristics is the network step, the more fine it is, the larger will be the angular separation at the output, while on the other hand a fine step is more difficult to manufacture.

These optical components allow to split a laser beam (from say a laser diode) to project a line, many lines, array of points (up to $19 \times 19$ ) and concentric circles (grid patterns should be available soon). These elements have many advantages such as no moving part, compactness, brightness uniformity (typ. better than 15\%), availability in different configurations such as separation angles, number of points, lines, circles projected, affordable cost (about $200 \$$ for a single line output head), etc. One problem is depth of focus of the output Powell lens used. To avoid say line blurring, Dammann networks must be operated at a larger than a minimum distance (although appropriate threshold of the Gaussian-shaped line section can circumvent the problem). Applications for which they are used include optical neural networks and of course machine vision (including TNDT).

\section{3DSS + TNDT: Case study}

In this section, we will briefly review a typical application of a 3DSS coupled to TNDT. As discussed in section 1, the purpose of this application is to enable thermographic inspection of large size components through robotic displacement of the TNDT head. The novelty of the approach lies in the self-navigation ability of the system which is described in [11]. Figure 10 shows a picture of the system. This system was built for demonstration purposes and stlll needs improvements before actual industrial implementation. It has two main limitations. First, the TNDT heating device is a $1600 \mathrm{~W}$ quartz infrared lamp with a back reflector only capable to stimulate efficiently low conductivity components such as plastic or CFRP panels. Second, the system works in two passes as described in the next paragraph. An improved system could require one pass only.

In the initial pass, the TNDT is turned off while the 3DSS (a Servo-Robot Jupiter camera) performs the ranging survey. Two lines of 256 ranging points are recorded (one at a time) and these points are associated three by three to form triangles whose normal is computed (Figune. 11). These orientations are used to determine whether or not the robot end-defector orientation and height above the surface are adequate. If correct, the robot pursues it: displacement, othenwise a correction is applied in order to maintain the perpendicular orientation of the head with respect to the scanned surface. After complete surface scanning, all the triangles are projected over a parametric model of the inspected surface. At this point, the observed surface is modeled and the system is ready for the second pass during which 
http://dx.doi.org/10.21611/qirt.1994.031

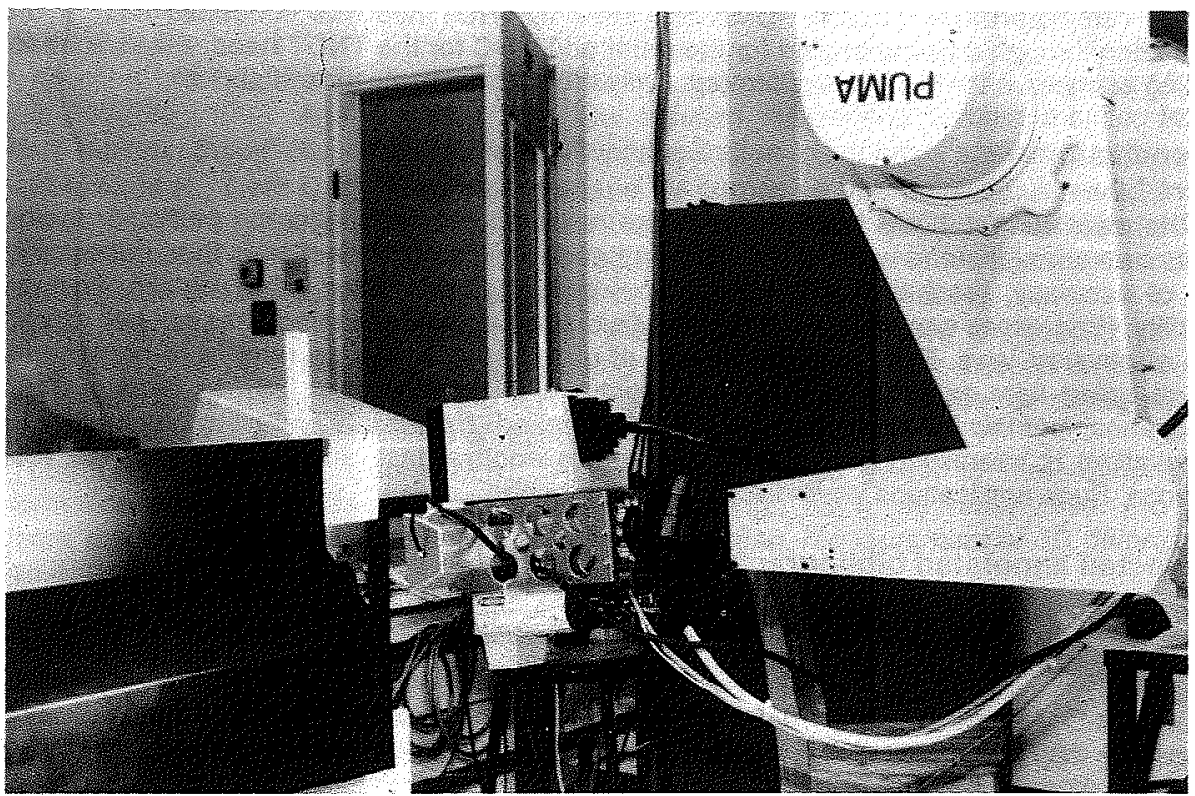

Fig. 10 - Case study: Picture of the apparatus for robotic TNDT inspection.

the ranging system is turned off and the TNDT inspection proceeds. Robot movements are based on the model of the surface recorded during the initial pass. From now on, a scanning strategy is required so that heat spreading from one field to the next is minimized. A line scanning thermal model was built for such purpose.

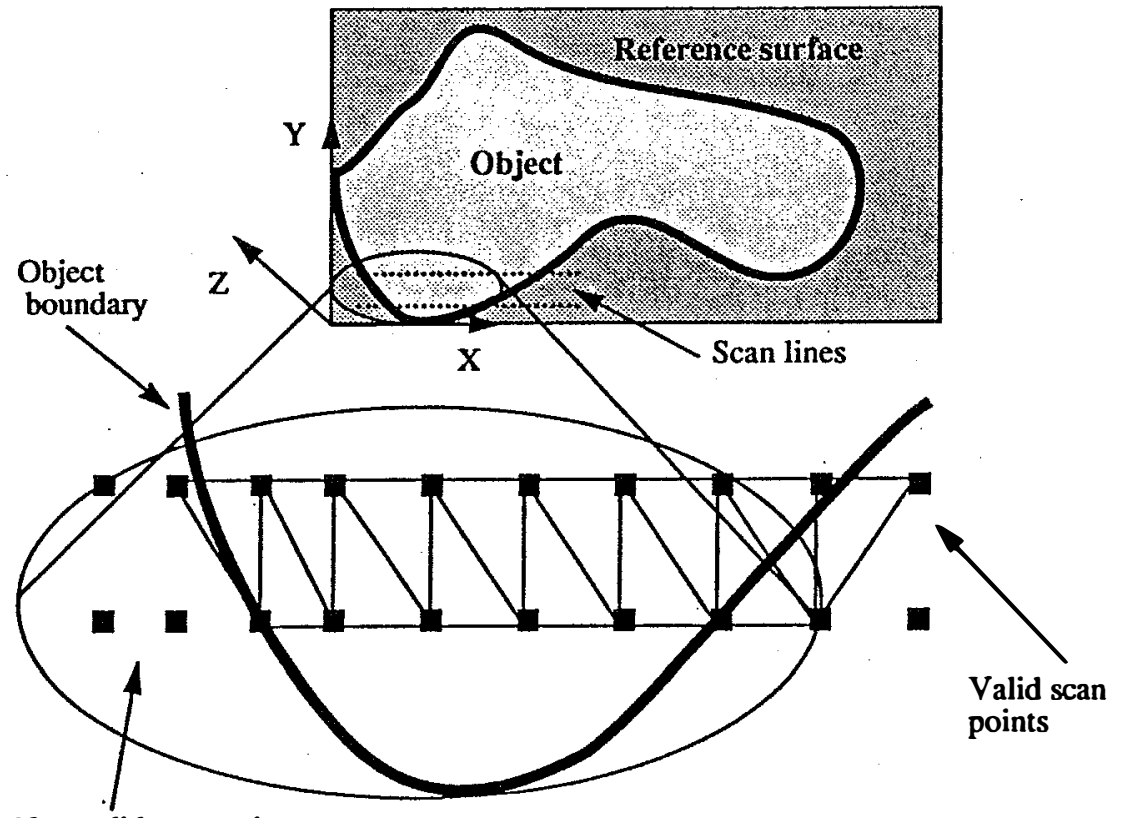

Non-valid scan points

Fig. 11 - Case study: Tridimensional scanning of the inspected surface (adapted from [11]). 
The complete system (robot PUMA 560 Mark II, TNDT head) is controlled from a Unix workstation through a Sparc engine for the robot and a PC for the TNDT head. Present work is ongoing to improve the current system (specially the limitations discussed above).

\section{Conclusions}

The main current 3DSS technologies were reviewed. As we saw, each particular method has its own strengths and weaknesses. When needed, a careful selection is thus necessary in term of accuracy, resolution, acquisition time, computational effort, size, weight, cost and reliability. Progresses can be expected in this field since actual technology has not yet reached limits of physical laws. For example, the use of observation in motion familiar to humans has not yet being fully exploited and new sensors are in development [28]. In term of TNDT applications, intrinsic 3D sensing is attractive but limited. Extrinsic 3D sensing (such as active triangulation) is interesting especially if coupled to another task such as for instance robot guidance. Such applications were discussed in the paper.

\section{Selected annotated bibliography}

In this section we present a selection of recent papers covering particularly interesting developments for the techniques reviewed in this paper.

- Brillault-O'Mahony B., "High level 3D structures from a single view," Image and vision computing, 10[7]:508-520, 1992. Perspective transformation to infer local configuration of features in $3 D$ space from a single view. Indoor scenes. Robot vehicle guidance.

- Bruce V., Coombes A., Richard R., "Describing the shapes of faces using surface primitives," Image and vision computing, 11[6]:353-363, 1993. Shapes, 2D pattems (surfacebase description, shape curvatures seen as primitives: peak, ridge, saddle ridge, minimal, pit, valley, saddle valley, flat.

- Cardillo J., Sid-Ahmed M.A., "3-D position sensing using a passive monocular vision system," IEEE trans. pattern analy. and mach. intellig., 13[8]: 809-813, 1991. Passive monocular 3-D position sensing made possible by a calibration scheme (robot) relating depth of focus blur through a composite lens and aperture model. Position accuracy is comparable to those of stereo techniques without correspondence problem.

- Cipolla R., Blake A., "Surface shape from the deformation of apparent contour," Int'I J. Computer Vision, 9[2]: 83-112, 1992. Local surface curvature is computed by spatiotemporal analysis of apparent contours in image motion. Robotics applications. Perspective projections.

- Crowley J. L., Stelmaszyk P., Skordas T., Puget P., "Measurement and integration of 3-D structures by tracking edge lines," Int'I J. Computer Vision, 8[1]: 29-52, 1992. Dynamic world modeling (predict, match, update) for 2-D appearance and 3-D geometry of a scene. Tracking of edge-lines in a sequence of images (camera mounted on a robot manipulator). Fusion of 3* $D$ data from different view points.

- Ermakov B.A., Voznitsky M.V., "Obtaining and processing information in pulsed laser range finders, Opt. Eng., 31[4]:768-781, 1992. Ranging object characteristics, permanent echo conditions, influence of target size.

- Goshtasby A., "Design and recovery of 2-D and 3-D shapes using rational gaussian curves and surfaces," Int'I J. Computer Vision, 10[3]: 233-256, 1993. Representation for parametrí curves and surfaces (2-D, 3-D). Control points of surface in this representation not required to form a regular grid (scattered set of control points sufficient).

- Hogg D. C., "Shape in machine vision," Image and vision computing, 11[6]:309-316, 1993. Review of common types of representations. Landmarks, parameterized and dynamic models, space warping.

- Hung D.C.C., "3D scene modeling by sinusoid encoded illumination," Image and vision computing, 11[5]:251-256, 1993. A sinusoidal illumination pattem is projected on the scene and surface structure is recovered by manipulating the deformation in the image. Reported $2 \%$ 
http://dx.doi.org/10.21611/qirt.1994.031

depth error. k-gradient operation to subdue influence of noise introduced in the process (differentiation).

- Karrati K., "3-D interpretation of optical flow by renormalization," Int'I J. Computer Vision, 11[3]: '?67-282, 1993. Analysis of optical flow induced by general camera motion. Image sphere representation. Biased noise model. Renormalization to suppress statistical bias.

- Kutulakos K., Dyer C.R., "Recovering shape by purposive viewpoint adjustment," Int'I J. Computer Vision, 12[2/3]: 113-116, 1994. Occluding contour method, motion (moving observer), viewing strategy to align viewing direction with one of the two principal directions for a point on the surface. No knowledge of velocities or accelerations required.

- Lee K.M., Jay Kuo C.C., "Shape from shading with perspective projection," CVGIP, 59[2]:2102-212, 1994. Shape from shading using perspective projection instead of commorly used orthographic projection not valid for objects close to camera. Model of object surface using iriangle patches. Linearization of reflectance map.

- Luo P.F., Chao Y.J., Sutton M.A., "Application of stereo vision to three-dimensional deformation analyses in fracture experiments," Opt. Eng., 33[3]: 981-990, 1994. Pin hole camera model, radial lens distortion taken into consideration, measurement of $3 D$ deformation of an object.

- Matsumoto T., Adachi M., Hayashi A., "Moirè topography for three-dimensional profile measurement using the interference fringes of a laser," Opt. Eng., 31[12]: 2668-2673. Interfenence fringes produced by a Mach-Zehnderinterferometer and a beam splitter produces both sets. Variable fringe distances (reported from 0.16 to $16 \mathrm{~mm}$ ).

- Ohya J., Kishino F., "Dense, time-varying range acquisition from stereo pairs of thermal and intensity images," in: Proc. Comput. vision patt. recogni., pp. 860-865, Seattle, June 2123, 1994. The stereo matching problem of correspondence between points in two intensity images recorded from different points of view is solved by using two thermal images which are non sensitive to view point change. Tilted mirrors allow to register each pair of visible and thermal' cameras. Dynamic programming allow to match isotemperature regions of themal images with corresponding intensity profiles in visible images to extract range. Real scenes.

- Rashid H.U., Burger P., "Differential algorithm for the determination of shape using a point light source," Image and vision computing, 10[2]:119-127, 1992. Recover shape information of Lambertian surfaces from the shading information inherent in a single $2 D$ image. Point light source close to the camera and near the surface (reflected light intensity function of the distance variation between the source and the image).

- Shih S.-W., Hung Y.-P., Lin W.-S., "Accurate linear technique for camera calibration considering lens distortion by solving an eigenvalue problem", Opt. Eng., 32[1]:138-149, 1993. Comparison with Tsai's calibration method. Low computational cost method. Camera parameters divided into two parts: lens distortion coefficient and combined geometric effects. Accuracy 1 part in 10,000 in 3D measurement.

- Soucy G., Ferrie F.P., "Motion and surface recovery using curvature and motion consistency," Report TR 93-14, MgGill Res. center for intell. machin., Mgill Univ., MontrËal (Canada) 29 pp., 1993. Algorithms to reconstruct surface shapes from overlapping range images using local motion estimates.

- Suganuma M., Yoshizawa, "Three-dimensional shape analysis by use of a projected grating image," Opt. Eng., 30[10]:1529-1533, 1991. Moirè contourgraphy, analysis with Fourier transform to detect shifted phases.

- Tan T.N., Baker K.D., Sullivan G.D., "3D structure and motion estimation from 2D image sequences, Image and vision computing, 11[4]:203-205, 1993. Two algorithms for depth estimation using point correspondences and ground plane constraint (permitted motion: translations along $x, y$ axis, rotation along $z$, z-axis points upwards). Occluded points accepted. Motion parameters extracted. Outdoor image sequences.

- Theodoracatos V., Calkins D.E., "A 3-D vision system model for automatic object surface sensing," Int'I J. Computer Vision, 11[1]:75-99, 1993. Vision system for object surface sensing. 3-D perspective and affine transformations, reconstruction process. Examples are given on a 
http://dx.doi.org/10.21611/qirt.1994.031

$20 \times 2$ ft rowing shell, average error. $0.2 \%$. Laser line projector to illuminate transverse sections of the object. Video camera.

- Watt R.J., "Issues in shape perception," Image and vision computing, 11[6]: 389: 394, 1993. Discussion about quality and suitability of particular shape representations and links with biological vision and manipulations.

- Wildes R.P., "Direct recovery of three-dimensional scene geometry from binocular stereo disparity," IEEE trans. pattern analy. and mach. intellig., 13[8]: 761-770, 1991. Recovery of 3-D orientation, discontinuities and relative distances. Texture geometry. Stability and reliability analysis. Natural images.

\section{Acknowledgments}

This work was supported by grants from Natural Sciences and Engineering Research Council of Canada, Fonds FCAR of the Quèbec province and by the Institute of Robotics and Intelligent Systems of Canada (member of the Canadian network of Centers of excellence).

\section{REFERENCES}

Note: see also additional references in section 8.

[1] VAVILOV (V.), TAYLOR (R.), "Theoretical and practical aspects of the thermal nondestructive testing of bonded structures," Research Techniques in NDT, R.S. Sharpe (ed.), 5, chap. 7: 238-279, 1982.

[2] CIELO (P.), MALDAGUE (X.), DĖOM-+ (A.), LEWAK (R.), "Thermographic NDE of Industrial Materials and Structures," Materials Evaluation, 45[12]: 452-460, 1987.

[3] BALAGEAS (D.L.), BOSCHER (D.), DĖOM (A.), FOURNIER (J.), HENRY (R.), "La thennographie infrarouge: un outil quantitatif à la disposition du thermicien," Rev. Gèn. Thenn. Fr., 322: 501-510, 1988, [in French].

[4] LAU (S.K.), ALMOND (D.P.), MILNE (J.M.), "A quantitative analysis of pulsed video thermography," NDT \& E Int'l, 24[4]: 195-202, 1991.

[5] MALDAGUE (X.), Nondestructive evaluation of materials by infrared thermography, Springer-Verlag, $224+16$ color pp, 1993.

[6] MALDAGUE (X.) (ed.), Infrared technology and methodology, Gordon and Breach Pub., 537 pp. (approx.), 1994, [in press].

[7] DAVID (D.), MARIN (J.Y.), DESSENDRE (M.), AVENAS-PAYAN (I.), LELIËVRE (B.), TRETOUT (H.), "SEQUOIA: Artificial intelligence applied to the infrared thennography inspection of composite aerospace structures," in Rev. Progress in Quantitat. NDE, Brunswick (Maine, USA), 8 p., August 1-6, 1993.

[8] VAVILOV (V.), MALDAGUE (X.), DUFORT (B.), IVANOV (I.), "Adaptive Thermal Tomography Algorithm," in: Proc. SPIE: Thennosense XV, L. R. Allen (ed.), 1933: 166-173, 1993.

[9] THEILEN (D.A.), CHRISTOFERSEN (R.J.), DODS (B.G.), EMAHISER (D.C.), ROBLES (B.H.), "Infrared thennographic inspection of superplastically fonned/diffusion bonded titanium structures," in: Proc. SPIE: Thennosense XV, L. R. Allen (ed.), 1933: 174-177, 1993.

[10] KRAPEZ (J.-C), BALAGEAS (D.), DĖOM (A.), LEPOUTRE (F.), "Early detection by stimulated infrared thennography. Comparison with ultrasonics and holo/shearography," in: Advances in Signal Processing for NonDestructive Evaluation of Materials, X. Maldague (ed.), NATO ASI Series, Kluwer Acad. Pub., E-262: 303-321, 1994.

[11] MALDAGUE (X.), PITRE (L.), MOUSSA (M.), LAURENDEAU (D.), "A robotic application of active thennographic NDT," to appear in Proc. 6th Eur. Conf. on NDT (Nice, France, Oct. 24. $28,94), 5$ p., [in press].

[12] SHIRAI (Y.), Three-dimensional computer vision, Springer-Verlag, 297 pp, 1987.

[13] BESL (P.J.), Surfaces in range image understanding, Springer-Verlag, 339 pp, 1989.

[14] SANZ (J.L.C.) (ed.), Advances in machine vision, Springer-Verlag, 420 pp, 1989. See in particular chapter 1 (pp. 1-63): "Active optical range imaging sensors" by P.J. Besl; chapter 2 (pp. 64-121): "3-D structures from 2-D images" by J.K. Agganwal and C.H. Chien; and chapter 
http://dx.doi.org/10.21611/qirt.1994.031

3 (pp. 122-159): "3-D sensing for industrial computer vision" by D. Poussart and D. Laurendeau.

[15] FREEMAN (H.), Machine vision for three-dimensional scenes, Academic Press, 419 p., 1990.

[16] HARALICK (R.M.), SHAPIRO (L.G.), Computer and Robot Vision, 2, Addison-Wesley, 630 Pp., 1993.

[17] MALDAGUE (X.), BARKER (E.), NOUAH (A.), BOISVERT (E.), DUFORT (B.), FORTIN (L.), "On methods for shape correction and reconstruction in thermographic NDT," in: Advances in Signal Processing for NonDestructive Evaluation of Materials, X. Maldague (ed.), NATO ASI Series, Kluwer Acad. Pub., E-262: 209-224, 1994.

[18] HORN (B.K.), Robot vision, MIT Press, 509 pp., 1986.

[19] LIU (T.-Y.), HSU (W.-H.), CHEN (Y.-S.), "Shading description via shading images," Image , and vision computing, 10[1]:46-54, 1992.

[20] GAGNON (H.), SOUCY (M.), BERGEVIN (R.), LAURENDEAU (D.), "Registration of Multiple range views for automatic 3-D model building," in: Proc. Comput. vision patt. recogni., pp. 581-586, Seattle, June 21-23, 1994.

[21] ZHENG (J.Y.), "Acquiring 3-D models from sequences of contours," IEEE trans. pattern analy. and mach. intellig., 16[2]: 163-178, 1994.

[22] BLAIS (R.), RIOUX (M.), "BIRIS: a simple 3D sensor," in Proc. SPIE Symposium on optics and optoelectronics advances in intelligent systems, 728: 183-194, 1986.

[23] RIOUX (M.), "Laser range finder based on synchronized scanners," Appl. Op., 23:38373844, 1984.

[24] BERALDIN (J.A.), RIOUX (M.), COURNOYER (L.), DOMEY (J.), "Registered intensity and range imaging at 10 mega-samples per second," Opt. Eng., 31[1]:88-94, 1992.

[25] CIELO (P.), Optical techniques for industrial inspection, Academic Press, 606 p., 1988.

[26] YEE (S.R.), GRIFFIN (P.M.), "Three-dimensional imaging system," Opt. Eng., 33[6]: 20702075, 1994.

[27] DAMMANN (H.), GÖRTLER (K.), "High efficiency in-line multiple imaging by means of multiple phase holograms," Opt. Comm., 3:312, 1971.

[28] GOSSELIN (C.), HAMEL (J.-F.), "The agile eye: a high performance three-degree-offreedom camera-orientating device," in Proc. IEEE Int'l conf. on Roboti. and Automat., San Diego, 781-786, 1994. 